\title{
AN INTEGRAL MEAN ESTIMATE FOR POLYNOMIALS
}

\author{
M. A. MALIK ${ }^{1}$
}

ABSTRACT. An inequality involving

$$
\max _{|z|=1}\left|P^{\prime}(z)\right| \text { and } \quad\left(\int_{0}^{2 \pi}\left|P\left(e^{i \theta}\right)\right|^{q} d \theta\right)^{1 / q}, \quad q>0,
$$

for a polynomial $P(z)$ having all its zeros in $|z| \leq 1$ is presented.

1. Let $P(z)$ be a polynomial of degree $n$ and $P^{\prime}(z)$ its derivative. Concerning the estimate of $\left|P^{\prime}(z)\right|$ when there is a restriction on the zeros of $P(z)$, Turan [9] proved the following: then

THEOREM A. If $P(z)$ is a polynomial of degree $n$ having all its zeros in $|z| \leq 1$,

$$
\frac{n}{2} \max _{|z|=1}|P(z)| \leq \max _{|z|=1}\left|P^{\prime}(z)\right| .
$$

The result is best possible and equality in (1) holds for $P(z)=\alpha z^{n}+\beta$ where $|\alpha|=|\beta|$.

In this paper we present a generalization of Theorem $\mathrm{A}$ in the sense that the left-hand side of $(1)$ is replaced by a factor involving the integral mean of $|P(z)|$ on $|z|=1$. In fact, our result is related to the following one of Saff and Sheil-Small $[8]:$

THEOREM B. If $P(z)$ is a polynomial of degree $n$ having all its zeros on $|z|=1$, then for each $q>0$

$$
\left(\int_{0}^{2 \pi}\left|P\left(e^{i \theta}\right)\right|^{q} d \theta\right)^{1 / q} \leq\left(A_{q}\right)^{1 / q}\left(\max _{|z|=1}|P(z)| / 2\right)
$$

where

$$
A_{q}=2^{q+1} \sqrt{\pi} \Gamma\left(\frac{1}{2} q+\frac{1}{2}\right) / \Gamma\left(\frac{1}{2} q+1\right) .
$$

The result is best possible and equality in (2) holds if and only if $P(z)=\alpha z^{n}+\beta$ where $|\alpha|=|\beta|$.

In the proof of Theorem B, Saff and Sheil-Small established the inequality

$$
n\left(\int_{0}^{2 \pi}\left|P\left(e^{i \theta}\right)\right|^{q} d \theta\right)^{1 / q} \leq\left(A_{q}\right)^{1 / q} \max _{|z|=1}\left|P^{\prime}(z)\right|
$$

for the polynomials $P(z)$ having all its zeros on $|z|=1$, and then in (3) used the Erdös-Lax theorem $[\mathbf{3}]$ to arrive at $(2)$.

Received by the editors March 23, 1982.

1980 Mathematics Subject Classification. Primary 30A10; Secondary 30C10.

Key words and phrases. Polynomials, derivative, zeros, inequalities.

${ }^{1}$ Research supported by NSERC, Canada. 
2. We now present our main result:

THEOREM 1. If $P(z)$ is a polynomial of degree $n$ having all its zeros in $|z| \leq 1$, then for each $q>0$

$$
n\left(\int_{0}^{2 \pi}\left|P\left(e^{i \theta}\right)\right|^{q} d \theta\right)^{1 / q} \leq\left(A_{q}\right)^{1 / q} \max _{|z|=1}\left|P^{\prime}(z)\right|
$$

where $A_{q}=2^{q+1} \sqrt{\pi} \Gamma\left(\frac{1}{2} q+\frac{1}{2}\right) / \Gamma\left(\frac{1}{2} q+1\right)$. The result is best possible and equality in (4) holds for $P(z)=\alpha z^{n}+\beta$ where $|\alpha|=|\beta|$.

REMARK 1. For a polynomial $P(z)$ having all its zeros in $|z|>1$, an inequality like (4) is not expected because the right-hand side of (4) may become arbitrarily small while $|P(z)|$ is uniformly close to 1 on $|z|=1$. This follows from a result due to MacLane [4]: for any $\varepsilon>0$ there exists a sequence of polynomials $T_{k}(z)$ having all its zeros on $|z|=1+\varepsilon$ and $T_{k}(z) \rightarrow 1$ uniformly on $|z| \leq 1+\varepsilon^{\prime}$ as $k \rightarrow \infty$, $\varepsilon^{\prime}<\varepsilon$. This observation makes Theorem 1 interesting.

REMARK 2. Making $q \rightarrow \infty$ in (4), one gets (1).

PROOF OF THEOREM 1. Let $P(z)$ be a polynomial of degree $n$. Define $Q(z)=$ $z^{n} \overline{P(1 / \bar{z})}$. It is immediate that $|P(z)|=|Q(z)|$ for $|z|=1$. Moreover, $P(z)=$ $z^{n} \overline{Q(1 / \bar{z})}$ so

$$
P^{\prime}(z)=n z^{n-1} \overline{Q(1 / \bar{z})}-z^{n-2} \overline{Q^{\prime}(1 / \bar{z})}
$$

from which

$$
z^{n-1} \overline{P^{\prime}(1 / \bar{z})}=n Q(z)-z Q^{\prime}(z) .
$$

Since $\left|z^{n-1} \overline{P^{\prime}(1 / \bar{z})}=\right| P^{\prime}(z) \mid$ for $|z|=1$, it further implies that

$$
\left|P^{\prime}(z)\right|=\left|n Q(z)-z Q^{\prime}(z)\right| \text {. }
$$

We also have

$$
\begin{aligned}
n|Q(z)| & =\left|n Q(z)-z Q^{\prime}(z)+z Q^{\prime}(z)\right| \\
& =\left|n Q(z)-z Q^{\prime}(z)\right||1+w(z)|
\end{aligned}
$$

where $w(z)=z Q^{\prime}(z) /\left(n Q(z)-z Q^{\prime}(z)\right)$. Hence for $|z|=1$,

$$
n|P(z)|=\left|P^{\prime}(z)\right||1+w(z)| .
$$

Next, we show that if $P(z)$ is a polynomial of degree $n$ having all its zeros in $|z| \leq 1$, then $w(z)$ is analytic in $|z| \leq 1$ and $1+w(z)$ is subordinate to $1+z$ for $|z| \leq 1$.

Since $P(z)$ has all its zeros in $|z| \leq 1$, by the Gauss-Lucas theorem $P^{\prime}(z)$ also has all its zeros in $|z| \leq 1$. This implies $z^{n-1} \overline{P^{\prime}(1 / \bar{z})}=n Q(z)-z Q^{\prime}(z) \neq 0$ in $|z|<1$. Moreover, if $|\alpha|=1$ is a zero of order $k(<n)$ of $n Q(z)-z Q^{\prime}(z)$, then from (5) it is also a zero of order $k$ of $P^{\prime}(z)$. As the unit disk which contains all the zeros of $P(z)$ is strictly convex, following the Gauss-Lucas theorem, $\alpha$ is a zero of order $k+1$ of $P(z)$ and so of $Q(z)$. Hence $\alpha$ is a zero of order $k$ of $Q^{\prime}(z)$. This concludes that $w(z)$ is analytic in $|z| \leq 1$. To show that $|w(z)| \leq 1$ for $|z|=1$ we note [5] that $\left|Q^{\prime}(z)\right| \leq\left|P^{\prime}(z)\right|$ for $|z|=1$. Also $w(0)=0$. Thus $1+w(z)$ is subordinate to $1+z$ for $|z| \leq 1$. 
Applying a well-known property of subordination $[\mathbf{2}]$ to (8) we deduce that

$$
\begin{aligned}
n\left(\int_{0}^{2 \pi}\left|P\left(e^{i \theta}\right)\right|^{q} d \theta\right)^{1 / q} & \leq \max _{|z|=1}\left|P^{\prime}(z)\right|\left(\int_{0}^{2 \pi}\left|1+w\left(e^{i \theta}\right)\right|^{q} d \theta\right)^{1 / q} \\
& \leq \max _{|z|=1}\left|P^{\prime}(z)\right|\left(A_{q}\right)^{1 / q} .
\end{aligned}
$$

This completes the proof of Theorem 1 .

3. By using the techniques in the above proof, we prove

THEOREM 2. If $P(z)$ is a polynomial of degree $n$, then

$$
\max _{|z|=1}\left(\left|P^{\prime}(z)\right|+\left|Q^{\prime}(z)\right|\right)=n \max _{|z|=1}|P(z)|
$$

where $Q(z)=z^{n} \overline{P(1 / \bar{z})}$. Moreover, the maximums of both sides in (9) are attained at the same point $\left|z_{0}\right|=1$.

Proof of ThEOREM 2. Let $P(z)$ be a polynomial of degree $n$. It is known [5] that for $|z| \leq 1$

$$
\left|P^{\prime}(z)\right|+\left|Q^{\prime}(z)\right| \leq n \max _{|z|=1}|P(z)| .
$$

We also note that from (5) and (6) one gets

$$
\begin{aligned}
n|P(z)| & =\left|z P^{\prime}(z)+n P(z)-z P^{\prime}(z)\right| \\
& \leq\left|P^{\prime}(z)\right|+\left|n P(z)-z P^{\prime}(z)\right|=\left|P^{\prime}(z)\right|+\left|Q^{\prime}(z)\right|
\end{aligned}
$$

for $|z|=1$. (10) and (11) imply (9). In view of (11), it is obvious that if $\left|P\left(z_{0}\right)\right|=$ $\max _{|z|=1}|P(z)|$ and $\left|z_{0}\right|=1$, then $\left|P^{\prime}\left(z_{0}\right)\right|+\left|Q^{\prime}\left(z_{0}\right)\right|=n\left|P\left(z_{0}\right)\right|$. This proves Theorem 2 .

If $P(z)$ is a self-inverse polynomial of degree $n$, i.e. $P(z)=z^{n} \overline{P(1 / \bar{z})}$, from Theorem 2 we get an already known result:

THEOREM C. If $P(z)$ is a self-inverse polynomial of degree $n$, then

$$
\max _{|z|=1}\left|P^{\prime}(z)\right|=\frac{n}{2} \max _{|z|=1}|P(z)| .
$$

Theorem C is proved by Govil [1], O'Hara and Rodrigues [6], and Saff and SheilSmall [8] independent of each other. In this case, we further note that $\left|P^{\prime}\left(z_{0}\right)\right|=$ $(n / 2)\left|P\left(z_{0}\right)\right|$, where $\left|z_{0}\right|=1$ and $\left|P\left(z_{0}\right)\right|=\max _{|z|=1}|P(z)|$. This implies that if $|P(z)|$ attains its maximum on $|z|=1$ at $n$ points ( $n$ is the degree of $P(z)$ ) then $\left|P^{\prime}(z)\right|$ must also attain its maximum at those $n$ points. Then $P^{\prime}(z)$ being a polynomial of degree $n-1$ must reduce to $P^{\prime}(z)=\alpha z^{n-1}$ where $\alpha$ is a constant. Consequently, we have

THEOREM 3. If $P(z)$ is a self-inverse polynomial of degree $n$ and $|P(z)|$ attains its maximum at $n$ points on $|z|=1$, then $P(z)=\alpha z^{n}+\bar{\alpha}$ where $\alpha$ is a constant.

REMARK 3. Rubinstein [7] has recently proved Theorem 3 for the class of polynomials having all its zeros on $|z|=1$. 
Finally, we give an application of Theorem $\mathrm{C}$ to real trigonometric polynomials. If $t(\theta)$ is a real trigonometric polynomial of order $n$, then

$$
e^{i n \theta} t(\theta)=R\left(e^{i \theta}\right)
$$

where $R(z)$ is a self-inverse polynomial of degree $2 n$. Differentiating (13) with respect to $\theta$ and applying Theorem $\mathrm{C}$, we get

$$
\max _{\theta} \sqrt{\{n t(\theta)\}^{2}+\left\{t^{\prime}(\theta)\right\}^{2}}=n \max _{\theta}|t(\theta)|,
$$

an inequality essentially due to van der Corput and Schaake [10].

We thank Professor Q. I. Rahman for useful discussions.

\section{REFERENCES}

1. N. K. Govil, On the derivative of a polymomial, Proc. Amer. Math. Soc. 41 (1973), 543-546.

2. E. Hille, Analytic function theory, Vol. II, Blaisdell, Toronto, 1962.

3. P. D. Lax, Proof of a conjecture of P. Erdös on the derivative of a polymomial, Bull. Amer. Math. Soc. 50 (1944), 509-513.

4. G. R. MacLane, Polynomials with zeros on a rectifiable Jordan curve, Duke. Math. J. 16 (1949), 461-477.

5. M. A. Malik, On the derivative of a polynomial, J. London Math. Soc. 1 (1969), 57-60.

6. P. J. O'Hara and R. S. Rodrigues, Some properties of a self-inverse polymomial, Proc. Amer. Math. Soc. 44 (1974), 331-335.

7. Z. Rubinstein, Personal communication.

8. E. B. Saff and T. Sheil-Small, Coefficient and integral mean estimates for algebraic and trigonometric polymomials with restricted zeros, J. London Math. Soc. 9 (1974), 16-22.

9. P. Turan, Ueber die ableitung von polymomen, Compositio Math. 7 (1939), 89-95.

10. J. G. van der Corput and G. Schaake, Ungleichungen für polymome und trigonometriche polymome. Compositio Math. 2 (1935), 321-361.

Department of Mathematics, Concordia University, Montreal (H3G 1M8), QUEBEC, CANADA 